\title{
Fictions du vide. Scénario pour une enquête cinématographique et littéraire
}

\author{
Thomas Carrier-Lafleur \\ Université Laval et Université Paul-Valéry (Montpellier III)
}

Exister, c'est différer.

Gabriel Tarde, Monadologie et sociologie

Comme l'indique son titre, le présent article proposera au lecteur de s'intéresser aux possibilités intrinsèques qu'ont les fictions cinématographiques et littéraires de "penser le vide", à travers un scénario qui en présente - dans une forme volontairement ouverte, et nécessairement incomplète - les différents acteurs et modes d'existence. Si l'on accepte l'axiome selon lequel nos défauts doivent devenir nos qualités, il est à souhaiter que les différentes connexions et correspondances 
testées au cours de ce texte ne soient pas prises par le lecteur pour ce qu'elles ne sont pas. Le vide et ses figures, la plus importante étant l'ellipse, ne sont pas le prétexte pour mener une réflexion épistémologique ou "archéologique», méthode qui a pourtant bien servi un penseur comme Foucault. Non, les limites de l'article en tant que forme, ajoutées au désir d'exploration qui nous habite, condamneraient à l'incomplétude la plus gênante une telle démarche, en dépit de son ostentatoire sérieux.

Sera plutôt mise en avant une autre forme d'incomplétude, celle-là consciente de sa nature et capable de se regarder dans le plus fidèle des miroirs : l'incomplétude ludique qui caractérise ce que nous croyons être une forme singulière de "gai savoir», c'est-à-dire l'enquête. Nous nous proposons donc d'enquêter sur le vide et ses figures, grâce aux concepts que sont à même d'instaurer un certain nombre de fictions préalablement choisies, selon le «casting » propre à l'enquête ou plutôt à son ébauche. Seront ainsi invitées à prendre place sur la scène des fictions cinématographiques et des fictions littéraires, dans cet ordre précis. Le cinéma, bien qu'il soit un art de l'enregistrement et de la projection, est aussi un art de l'absence, d'abord en ce qu'il ne peut que renvoyer les images de corps qui ont vécu dans un autre espace et dans un autre temps. Il existe donc un rapport étroit entre le cinéma et le vide, comme le dit bien un récent ouvrage qui a aussi l'avantage de favoriser une méthode "kaléidoscopique», en opposition à cette pesanteur de la pensée critique - colosse de rigueur qui parfois oublie ses pieds d'argile. Nous parlons du beau texte de Jean-Louis Leutrat sur le «fantastique du cinéma », Un autre visible. Sans incarner une nouvelle panacée, ce livre a au moins le mérite de réfléchir, dans une forme qui s'accorde avec son contenu, sur ce que l'auteur nomme 
«l'invisible relatif » qui caractérise un certain mode d'existence du cinéma. Le film, en lui-même, est un " objet fantomatique », une manifestation du vide, puisqu'il est pour le moins complexe d'affirmer qu'il existe bel et bien en dehors de sa projection. De ce point de vue, que nous espérons avoir fait nôtre, l'art cinématographique a cette particularité de mettre facilement l'accent sur la dimension performative du vide. C'est ce qui nous intéressera lorsque nous proposerons une sorte de polémique fictive - que le lecteur ne doit pas lire comme le fin mot de l'histoire, mais répétons-le comme une ébauche ou un scénario - entre Gilles Deleuze et André Bazin (ce dernier jouera pour ainsi dire le rôle rhétorique du «vilain », malgré la grande force de sa pensée, que nous sommes bien sûr les premiers à reconnaître). La pensée « en rhizome » de Deleuze nous permettra de déployer de nouvelles connexions et ainsi de transporter notre enquête vers les terres de la littérature. L'instauration littéraire du vide ne s'oppose pas à son équivalent cinématographique, bien au contraire. C'est pour cela que nous y porterons une attention particulière, afin de proposer au lecteur - de qui nous souhaitons la participation active à notre jeu - une façon de voir en quoi les figures du vide forment un réseau essentiellement interdisciplinaire.

Le vide n'est jamais que du vide. Derrière son passage, il laisse un nombre impressionnant de traces, que l'enquête se doit de relever afin de construire son scénario. Le lecteur ne doit pas prendre des vessies pour des lanternes, en croyant que notre scénario vise aveuglément une autonomie ou une matérialité. À l'inverse, en dépliant nos exemples d'instaurations fictionnelles, qu'elles soient cinématographiques ou littéraires, et en suivant les traces des figures du vide, nous visons une forme de vérité qui relève du relativisme 
en tant que "vérité de la relation », tel que le pense justement Deleuze dans son ouvrage sur Leibniz et le baroque. Notre scénario, "baroque » lui aussi, sera préférablement lu comme une poussée vers l'abstraction, ce qui offre l'avantage de correspondre au sujet principal de l'enquête.

\section{Position fictionnelle d'un problème : les acteurs}

Il existe au cinéma un certain nombre de concepts que l'on attribue plus volontiers non pas à un réalisateur qui en a fait l'usage, mais à un théoricien qui, après coup, en a proposé la description la plus poussée ou la plus caractéristique. Le «montage interdit» d'André Bazin est de ceux-là. Il s'agit par ailleurs d'un élément important de son «ontologie» cinématographique ${ }^{1}$. Même sommairement, comment faut-il qualifier cette science de l'être? Peut-être devrait-on d'abord mentionner qu'il s'agit d'une ontologie réaliste et, par là même, positive, n'étant pas fondée sur le vide, mais sur le plein : l'actuel y règne sur le virtuel, ce qui est vu sur ce qui est absent, le dit sur le suggéré, etc. Certains ont par ailleurs qualifié non sans raison le montage interdit de « loi de Bazin » (Deleuze, p. 211).

Bazin critique le recours excessif au montage en ce qu'il n'est qu'une «création abstraite de sens ». À première vue, ce retournement a de quoi surprendre: longtemps compris

\footnotetext{
${ }^{1}$ Le lecteur aura compris que nous jouons ici le jeu - dangereux s'il en est qui consiste à lire Bazin comme un « métaphysicien », dialectisant le paraître et l'apparaître cinématographiques. Cette position du critique en philosophe, que nous n'endossons pas totalement, est néanmoins profitable pour la qualité de notre scénario, ce pour quoi nous la conservons malgré d'éventuelles contestations.
} 
comme ce qui était essentiellement cinématographique dans l'art du cinématographe, le montage devient «le procédé littéraire et anti-cinématographique par excellence». En contrepartie, "[1]a spécificité cinématographique, saisie pour une fois à l'état pur, réside au contraire dans le simple respect photographique de l'unité de l'espace» (Bazin, p. 55). Vision réductrice du cinéma, diront certains? On pourrait éventuellement leur donner raison, mais pas sans avoir noté ceci : si la vision de Bazin est d'une façon ou d'une autre « réductrice », c'est qu'elle vise d'abord à mettre l'accent sur le positivisme de l'art cinématographique. Cela revient à dire que le théoricien se présente - peut-être malgré lui - comme un acteur qui privilégiera toujours ce qui est vu à ce qui est suggéré, ce qui est effectivement dit à ce qui est caché dans les «blancs » du montage.

Du même coup, une voix s'élève, et une opposition est officiellement formulée. Notre enquête doit en prendre note, la littérature se trouve ici taxée de subterfuge : il lui manquerait la "plénitude esthétique» (le terme est de l'acteur Bazin) de l'entreprise soi-disant réaliste du cinéma. Art de la continuité, d'une vision $\mathrm{du}$ monde toute positive, la forme cinématographique décrite par Bazin dans "Montage interdit » (nous ne disons évidemment pas que cela soit le cas dans tous ses articles) se trouve ainsi dans la position de juge et partie: elle frêne ses ardeurs, en imposant certaines limites à la pratique même du montage. Pour être bien compris, on proposera cette formule toute manichéenne, qui fait de Bazin le «méchant» dont notre scénario a besoin : le montage interdit demande l'absolue simultanéité des divers facteurs d'une action déterminée. Et Bazin de proposer l'exemple célèbre du Cirque (1928) de Chaplin, où ce dernier se retrouve dans la même cage 
qu'un lion supposé féroce : pour « croire » à la réalité de cette action, au moins un plan devra nous montrer Chaplin en compagnie du dangereux animal. Le même phénomène peut s'observer lors de l'enregistrement d'une performance sportive, ou encore d'un spectacle de magie. Au contraire, aussi véridique que se présente un récit littéraire (même autobiographique), un doute raisonnable peut et devra rester dans la tête du lecteur. Si la continuité littéraire n'est qu'artifice, au sens où Bazin entend ce mot, c'est qu'elle n'est que montage.

À l'inverse, la reproductibilité technique sur laquelle s'assoit le cinéma lui permet un rapport direct aux multiples facteurs qui font la continuité de l'événement, même si celui-ci est capté dans les conditions «truquées » du plus hollywoodien des studios. Un comédien que l'on voit « réellement » suspendu dans le vide, c'est-à-dire sans découpage des facteurs particuliers de cette action (main agrippée au précipice, goutte de sueur, rictus paniqué, vide sous les pieds du comédien, etc.), est bien entendu protégé par un filet de sécurité, que la caméra aura la décence de ne pas inclure dans son champ. Avec les diverses technologies du numérique que nous connaissons et pratiquons aujourd'hui, les gros traits de cette «loi de Bazin » (que nous avouons certes avoir quelque peu réécrite pour servir la suite de notre enquête) se trouvent plus que jamais remis en question. Il n'est pas rare que des comédiens jouent devant des "écrans verts » sur lesquels sera juxtaposé un espace qui se veut réel, surface où viendront virtuellement s'ajouter les facteurs déterminants de l'action.

Mais était-il nécessaire d'attendre de pareilles avancées techniques pour relativiser les principes de ce «montage interdit» en tant que pierre de touche cinématographique? 
Évidemment pas. L'histoire du cinéma n'est pas dogmatique, si bien que la théorisation de la simultanéité des facteurs, telle que l'on vient de la décrire, s'accompagne d'une entreprise de dislocation de l'action: une contestation de la continuité spatiale et temporelle (Chaplin partageant le même espacetemps que le lion, Harold Lloyd s'agrippant de façon précaire après une horloge géante dans Safety Last, etc.). C'est par exemple ce que Deleuze remarque lorsqu'il développe la grande et la petite forme de "l'image-action», enjeux qui seront appelés à devenir des éléments constitutifs de notre enquête sur les figures et les fictions du vide.

\section{Découverte des traces : la grande et la petite forme}

Cette opposition entre la " grande forme » et la «petite forme » cinématographique est entièrement redevable à Deleuze. Notre enquête nous amène ainsi à rencontrer un autre acteur, donnant ainsi une nouvelle couleur à notre ébauche de scénario. Dans le même temps, ces deux concepts deleuziens seront importants, essentiels même, afin de poursuivre notre examen des traces laissées sur leur chemin par les figures du vide. La grande et la petite forme, telles que nous les comprenons, représentent moins une dialectique parfaite (et donc fermée) qu'une mise en scène de deux modes d'instauration propres à deux catégories de fictions. En tentant de conserver le «ton » propre au langage du philosophe, nous présenterons au cours des lignes qui suivront notre interprétation de cette mise en scène conceptuelle. Le lecteur cherchera donc inutilement son lot de citations explicatives, puisque cette méthode est contraire à notre intuition : on ne 
cite pas dans un scénario, on tente d'offrir la description la plus piquante possible des notes que l'on aura pu recueillir lors de l'enquête.

Pour définir la grande forme cinématographique, un exemple souvent utilisé par Deleuze est celui du western : la séquence du duel est bien construite sur le montage et plus encore sur le montage d'action, mais il faut bien qu'à un moment ou à un autre soient présentés les deux personnages en un seul et même plan, mettant ainsi en avant l'unité de lieu et de durée. Plus encore, une des principales caractéristiques du cinéma de la grande forme réaliste est de se déployer dans un espace qui vaut pour lui-même, un espace "actualisé », avance Deleuze au cours du neuvième chapitre de Cinéma 1. Du milieu émanent des «lignes de force» ou encore des «foyers » qui influencent directement le personnage, par exemple les bandits qui menacent l'autorité du shérif d'une petite ville. Un ensemble d'actions converge ainsi vers le personnage et tend à modifier la situation initiale, en vue d'une nouvelle situation: avant et après un duel. La formule de la grande forme cinématographique serait donc " $S-A-S^{\prime}$ »: situation-action-situation nouvelle. Le rôle du montage est ici d'agencer le rapport de ces lignes de force afin de monter en intensité la situation au point où l'action devient nécessaire. Et c'est précisément à ce moment que le «montage interdit » refait surface : il érige en tant que loi la simultanéité de tous les facteurs déterminés qui sont sortis de la situation et du milieu, et permet l'arrivée de l'action qui porte avec elle la charge d'une situation nouvelle.

Le cinéma de la petite forme renverse la formule. De «SAS" », on passe à «ASA' ». La situation est cachée au spectateur : n'en seront dévoilés que des morceaux, à partir des 
différentes actions du film. On passe d'un cinéma de l'actuel aux puissances de la virtualité : un cinéma du non-dit, du vide, de l'indice et de l'ellipse. Cette autre modalité des fictions cinématographiques se rapproche donc de ce que l'on a appelé « ontologie négative» (Nef, p. 19). Nous lirons ainsi la petite forme en tant qu'art des ellipses. L'action perd de son efficace : elle est réduite au simple fait de montrer un morceau ou un pan de la situation, qui en tout point la dépasse, et ainsi elle suggère la nécessité d'une action nouvelle pour éclairer une autre dimension de la situation. Le cinéma de la petite forme convoque ainsi la participation active du spectateur, ce dernier devant combler les «blancs » du récit et reconstituer pour luimême toute l'incommensurabilité de la situation. Avec la figure de l'ellipse des fictions de la petite forme, la réalité se transforme en signes. La situation n'est plus ce milieu actuel où sont montées en puissance les lignes de force jusqu'au climax de la simultanéité des facteurs d'une action déterminée, elle devient plutôt le chaînon manquant qui bloque la continuité entre les actions des personnages.

L'enquêteur peut donc valider une intuition antérieure : la situation incommensurable des fictions du vide est analogue à la dislocation de l'action précédemment évoquée. De façon métaphorique, la petite forme donne à voir sans pour autant montrer. D'ellipse en ellipse, le spectateur accumule les indices qui sont autant de points de vue sur la situation, essentiellement changeante, du récit. Pour paraphraser Deleuze, on pourrait avancer que ne sera connu de la situation que ce qu'en laisse deviner l'action en tant que marque ou qu'indice, d'où le rapport de la petite forme avec ce que nous appelons une enquête. 


\section{Deux modes de la "petite forme » : Lubitsch et Nichols}

Dans le cinéma parlant, les « sex comedies » de Lubitsch forment sans doute l'un des corpus qui a le plus exploité la dimension comique de la petite forme. D'abord par l'utilisation qui y est faite de la parole, qui n'est pas tant illustrative que suggestive. Le montre bien un film comme To Be or Not to Be (1942; Jeux dangereux, dans sa traduction française), où la fameuse tirade shakespearienne est privée de sa grandeur poétique au profit d'un détournement riche en sous-entendus: lors d'une représentation de la pièce, elle devient le signal qui indique au Lieutenant Stanislav Sobinski (Robert Stack) qu'il est l'heure d'aller rejoindre dans sa loge sa maîtresse et comédienne Maria Tura (Carole Lombard), épouse de l'acteur Joseph Tura (Jack Benny). Ce dernier, étant sur scène en train de réciter le fameux monologue, ne peut se douter de l'ampleur que prend sa situation conjugale. Selon les dires de Lubitsch, un tel procédé de déviation - suggérer l'essentiel plutôt que de le montrer aurait pris forme dans un film précis de Chaplin (où son personnage n'apparaît d'ailleurs pas): The Woman of Paris (1923; L'Opinion publique). À la suite d'un malentendu amoureux et familial, une pauvre jeune femme aux traits quasi asexués quitte subitement la province et l'homme qu'elle devait épouser pour se rendre à Paris. Ellipse de plus ou moins une année, et le spectateur la retrouve belle, sensuelle et, découvrira-t-on assez rapidement, maîtresse du plus riche célibataire de la Ville lumière. Toute la seconde partie du film le jeune homme anciennement aimé se rend à Paris pour retrouver la jeune femme - exploite ce vide narratif, par indices interposés. Il y a ainsi un parallélisme entre le personnage de l'homme (de province) et le spectateur. Tous 
deux sont désavantagés face à la situation. Ils doivent procéder par déduction pour tenter d'en reformer le puzzle. Deux moments dans le film sont célèbres à cet égard. D'une part, le départ pour Paris, où le train ne sera jamais montré, mais seulement suggéré par son ombre sur le visage de la jeune femme, soulignant du même coup le destin "funeste» qui l'attend (Chaplin a en effet sous-titré son film «A Drama of Fate »). D'autre part, la scène où le jeune homme, dans la chambre de son ancienne compagne, voit par terre un faux col, découvrant par cet indice la présence d'un amant dans la vie de la jeune femme qu'il croyait encore plus ou moins innocente.

Notre enquête nous amène maintenant à constater la radicale absence de psychologie dans ces films, comme si la découverte de l'indice avait d'abord et avant tout une valeur en soi. Les figures du vide s'y installent comme des motifs autosuffisants. Certes, le cinéaste force son spectateur à comprendre les moindres soupirs qui rythment ses comédies, mais l'opération de déduction ne trouve pas sa fin à l'extérieur d'elle-même. C'est ce que montre de façon métaphorique Trouble in Paradise (1932; Haute pègre), histoire d'un couple de bandits commettant larcin après larcin, sans que ne soit jamais montré un seul et unique vol. À l'inverse de ce procédé, se trouve un film comme Pickpocket (1959), dans lequel Robert Bresson accorde une attention démesurée aux mains des malfrats et au subtil circuit des portefeuilles volés. Chez Lubitsch, l'action est toujours disloquée, car le vol prend forme dans l'ellipse: il n'est évoqué qu'après coup par le transfert spatial des objets. Par ailleurs, dans cette redistribution aussi improbable qu'audacieuse des objets, le film se montre comme film. La petite forme est en effet un moyen par lequel le cinéma met l'accent sur sa nature spéculaire. L'ellipse se donne à 
comprendre comme "truc » (au sens de Méliès), comme artifice. Le film affiche ses coutures ou, plutôt, ses blancs. Le spectateur s'intègre au flux du film, voire au film en tant que film (cf. Durand, 1993). C'est en faisant l'expérience du manque propre à ces fictions du vide que le spectateur semble le plus créatif : l'instauration éthique et esthétique de telles œuvres le transforme en enquêteur. Se trouvent à nouveau validées nos intuitions quant à la forme que se devait d'adapter le présent texte, afin d'être en adéquation avec son contenu.

Cela dit, quel serait l'autre pôle de la petite forme? Dans les fictions de Lubitsch, le circuit des indices débouche sur une sorte de joyeuse absurdité ou sur la connivence intrinsèque entre le réalisateur et le spectateur. Se crée ce que Deleuze nomme, en s'inspirant ouvertement de Bachelard, un « cristal » où l'action se disloque : un plan où les marques et les indices, en gagnant en autonomie, accentuent la nature spéculaire de l'œuvre. Les figures du vide sont ainsi responsables de la communication virtuelle entre la fiction et le spectateur. Mais qu'advient-il lorsque le cristal se «fêle »? Que se passe-t-il, par exemple, entre le cristal en apparence parfait et accueillant des comédies loufoques de Lubitsch et les drames de mœurs signés Mike Nichols (cinéaste qui, toutes proportions gardées, nous semble reprendre pour son compte les spécificités de la «touche» des fictions lubitschiennes)? On peut se risquer à formuler l'hypothèse suivante: il s'insère une quantité non négligeable de «fissures » au sein de la fiction, ce qui a pour effet de souligner une certaine limite de la petite forme. En un mot : le comique devient tragique.

C'est ce que montrent les premières images de Closer (2004; Entre adultes consentants). Le refus de la simultanéité 
des facteurs constitutifs de l'action ne débouche plus seulement sur une redistribution disloquée de l'action, mais la situation incommensurable se définit maintenant par ce qu'elle a d'abominable et de pathétique. Cette scène, uniquement construite en rapides champ-contrechamps, montre d'un côté une jeune femme (Nathalie Portman) et, de l'autre côté, un jeune homme (Jude Law) marchant dans une rue bondée. Dans leur cadre respectif, les personnages sourient. Peut-il s'agir de la même rue grisâtre? Se regardent-ils? Si oui, se reconnaissentils ou alors est-ce un début de flirt? Ils regardent à droite, puis à gauche, c'est-à-dire vers deux hors-champs, deux espaces vides où se multiplient les possibles. La jeune fille regarde une dernière fois à droite, puis on voit le regard apeuré du jeune homme. L'inventivité presque scandaleuse, mais toujours pleine de vie, des films de Lubitsch fait ici place à un certain effondrement de la fiction qui est comme le symbole d'une décomposition propre à la petite forme cinématographique. Suivant la formule deleuzienne "ASA" », le plan suivant a sauté la situation incommensurable : l'accident, bien évidemment.

Du point de vue narratif, cet accident est mineur, en ce sens que la jeune fille ne sera que légèrement blessée, mais d'un point de vue métaphorique, il soulève l'altération de la petite forme lorsque celle-ci va du comique au tragique: dès ses premiers moments, la fiction met en scène une fêlure et rend impossibles les jeux de l'amour et du hasard. Par la suite, le film remplacera la logique du triangle amoureux, toujours jovial chez Lubitsch, par les va-et-vient répétitifs et quelque peu sordides entre deux couples. De la chaude légèreté comique on passe au caractère gelé, anesthésié presque, du tragique. Des petits gestes, tous plus banals les uns que les autres, souligneront d'autres fêlures, feront comprendre aux 
spectateurs qu'un autre adultère vient d'être commis, qu'une autre rechute vers les pulsions destructrices a déjà eu lieu. Le spectateur n'a même plus besoin de voir, il sait que la situation ne peut aller que de mal en pis, rappelant du même coup qu'il ne faut pas badiner avec les figures du vide.

Dans un cinéma comme celui de Lubitsch, la rapidité rendue possible grâce à la parfaite maîtrise des ellipses était comme un défi lancé au spectateur qui devenait par là aussi joueur que les personnages de la comédie. Alors que, dans le tragique de Nichols, chaque nouveau vide est le signe d'un autre stade dans la décomposition morale des personnages du drame. Si l'on interrogeait Deleuze, il répondrait sûrement que le circuit des indices devient la ronde de la fêlure des comportements, des mœurs, des habitudes, comme si le jeu venait de se fermer sur lui-même. Dans l'œuvre de Lubitsch, on ne cesse de sortir du cristal, grâce à la force du désir ou de la curiosité ludique. À l'inverse, avec le cinéma de Nichols, on s'enfonce de plus en plus au cœur du cristal, une fois que celuici s'est fissuré. La dislocation de l'action poursuit sa route jusqu'au cœur du cristal de la fiction.

Des nouveaux territoires que notre enquête nous a amené à fréquenter, certaines questions émergent. D'où vient cette oscillation entre les deux côtés de la petite forme? L'ellipse comique et l'ellipse tragique sont-elles foncièrement différentes? Avec un cinéaste comme Lubitsch, chaque découverte d'indice était comme un défi lancé au spectateur. L'originalité de telles fictions consistait entre autres dans leur utilisation du scepticisme en tant que mode d'expression joyeux. Des pensées nouvelles prennent forme grâce à la mise en scène du vide. Y est aussi engendrée une restructuration 
temporelle où le quotidien des personnages se transmue progressivement en expérience privilégiée, celle de la déduction. Pour reprendre un commentaire de Marc Cerisuelo (2012, p. 194), «les limites de notre connaissance ne constituent en rien un défaut de notre faculté de connaître ». Les vides qui rythment les fictions lubitschiennes sont l'occasion pour les personnages d'expérimenter, et si possible de rapetisser, l'écart qui les sépare. À l'inverse, on l'aura compris, l'intervalle qui sépare les personnages dans le mode tragique de la petite forme est un écart en constant agrandissement. Dans les tragédies de la petite forme, le présent passe sans que les personnages puissent l'attraper ou en faire quelque chose, à l'instar de l'accident qui ouvre Closer.

Pour conclure sur la différence qui est en mesure de s'installer entre les deux modes d'existence des fictions du vide - d'une part le manque joyeux et d'autre part le vide fêlé -, on peut s'amuser à rappeler cette blague racontée par un personnage de Ninotchka (1939) : «Un homme entre dans un restaurant. Il s'assoit et demande au serveur un café sans crème. Cinq minutes plus tard le serveur revient et dit, "je suis désolé Monsieur, nous n'avons plus de crème. Pourrais-je vous servir plutôt un café sans lait?" ». L'ébauche d'enquête que nous sommes en train de livrer ne vise pas autre chose qu'une telle prise de conscience : il n'est pas adéquat de définir les êtres et les figures de fiction comme de simples produits de l'imagination. En effet, le personnage du film de Lubitsch a bien raison de remarquer qu'il y a une différence entre le café sans crème et le café sans lait, puisque le lait et la crème sont deux agents porteurs de scénarios différents. La commande d'un café sans lait est bel et bien différente de celle d'un café sans crème, à l'instar des figures du vide dans les deux pôles de la petite 
forme. La problématique qu'installent ces différentes figures vides met donc l'accent sur la souplesse interprétative qu'il faut posséder pour dialoguer avec les êtres de fictions. Entre le manque de crème et le manque de lait, il y a une différence de nature.

\section{Reprise littéraire de la problématique : de nouveaux acteurs}

Il faut maintenant revenir à la promesse faite au lecteur au début de ce texte, celle d'une enquête intermédiatique. Cela ne fera pleurer personne, mais il faut bien le reconnaître: le cinéma n'est pas le seul art capable de créer des fictions du vide. La littérature, bien que des raisons évidemment techniques l'empêchent de jouir de la dialectique de la projection et de l'absence, était avant le cinéma un art de l'ellipse. Parfois, un récit va même être célèbre non pas pour ce qu'il dit, mais bien pour ce qu'il ne dit pas ou ne fait qu'évoquer. On peut penser à l'exemple de Proust lecteur de Flaubert :

Je ne me lasserais pas de faire remarquer les mérites, aujourd'hui si contestés, de Flaubert. L'un de ceux qui me touchent le plus parce que j'y retrouve l'aboutissement de modestes recherches que j'ai faites, est qu'il sait donner avec maîtrise l'impression du Temps. À mon avis la chose la plus belle de L'Éducation sentimentale, ce n'est pas une phrase, mais un blanc. Flaubert vient de décrire, de rapporter pendant de longues pages, les actions les plus menues de Frédéric Moreau. Frédéric voit un agent marcher avec son épée sur un insurgé qui tombe mort. «Et Frédéric, béant, reconnut Sénécal! » Ici un «blanc», un énorme «blanc » et, sans l'ombre d'une transition, soudain la mesure du temps devenant au lieu de quarts d'heure, des années, des décades. (1975, p. 595)

On se souvient du passage en question :

Il voyagea. 
Il connut la mélancolie des paquebots, les froids réveils sous la tente, l'étourdissement des paysages et des ruines, l'amertume des sympathies interrompues.

Il revint. (1989, p. 509)

Comme le cinéma, la littérature est un terrain d'enquête privilégié pour retrouver les figures du vide et pour questionner leur portée morale et esthétique. L'exemple de Proust serait fascinant à étudier sur ce point, ne serait-ce que par la figure du narrateur, ce personnage duquel on connaît à la fois tout et rien, à commencer par son portrait physique : le « je » d'À la recherche du temps perdu peut en effet se comprendre comme une sorte de vide ambulant, une multitude non localisable d'organes sans corps. Il observe, sent, aime, touche, sans que ces sensations puissent se rapporter à un espace-temps déterminé. La phrase de Proust permet au spectateur de parcourir en une seule ligne plusieurs lieux, de vivre plusieurs durées, sans que ces expériences se rapportent à une figure romanesque spatialement et temporellement repérable. L'action proustienne est l'action disloquée par excellence.

Lorsqu'on étudie le rapport de coexistence entre les arts et les modes d'expression, une règle devrait tenir : servons-nous de tout. Détournons-nous donc un instant de la littérature, afin de mieux y revenir, et retrouvons l'acteur principal de ce scénario. Il est en effet passionnant de mettre l'accent sur l'ossature conceptuelle utilisée par Deleuze pour former la dialectique entre la grande et la petite forme ou, pourquoi pas, l'épique et l'elliptique ${ }^{2}$. Dans son étude comparée des deux

2 On renverra le lecteur au onzième chapitre de Cinéma 1. L'Imagemouvement, «Les figures ou la transformation des formes », particulièrement à la troisième partie de ce chapitre, et plus encore au cours sur le cinéma donné par Deleuze à l'Université Paris-VIII le 27 avril 1982, disponible sur Internet à l'adresse suivante: www2.univ-paris8.fr/deleuze/. On soulignera 
formes, Deleuze utilise trois niveaux d'analyse : épistémologique, mathématique et esthétique. Pour développer quelque peu cet exemple précis, le philosophe porte d'abord son attention sur l'évolution de la notion scientifique de milieu, principalement grâce aux travaux de Canguilhem, de Lamarck et d'Auguste Comte. Quel rapport avec les formules «SAS' » et « ASA'»? Sans entrer dans les détails, on voit d'emblée que le terme médiateur n'est pas le même : soit une action entourée de situations, ou l'inverse. Ces deux formes d'expression, peuton se risquer à avancer, représenteraient du même coup deux visions de l'homme dans sa relation avec le monde. Dans la grande forme, le milieu est «bio-anthropologique » et, avec la petite forme, «physico-biologique» (Deleuze, p. 253). Suivant la logique de la formule "SAS' », l'homme est au centre des choses, il est entouré de milieux, de "situations », comme nous disions plus tôt. L'action catalyse les forces et permet la succession des situations, la transformation des milieux. Pour reprendre notre exemple précédemment évoqué : la ville du western classique avant et après le duel entre le shérif et le dangereux cowboy. Le milieu agit sur le vivant et, à son tour, le vivant réagit sur lui. Les milieux sont actualisés, déterminés. On peut les qualifier: la ville où règne le chaos, la ville où règne l'ordre. La petite forme possède cette différence notable que c'est justement le milieu qui est au milieu, c'est-à-dire le milieu en tant qu'intermédiaire.

Nous laissons à la curiosité du lecteur la possibilité de s'informer lui-même sur la dimension mathématique des formes cinématographiques. Toutefois, il n'est sans doute pas

aussi à ce propos le récent livre de Jean-Michel Pamart : Deleuze et le cinéma. L'armature philosophique des livres sur le cinéma. 
déplacé de nous intéresser au domaine esthétique de la surprenante généalogie conceptuelle esquissée par Deleuze. Le philosophe met en relief les analogies entre le cinéma et la peinture chinoise et japonaise, à partir de la pensée de l'écrivain et historien de l'art du VIe siècle Sie Ho. Deleuze écrit : « d'une part le vide primordial, et le souffle vital qui imprègne toutes choses en Un, les réunit en un tout et les transforme suivant le mouvement d'un grand cercle ou d'une spirale organique; d'autre part le vide médian, et l'ossature, l'articulation, la jointure, ride ou trait brisé, qui va d'un être à un autre en les prenant au sommet de leur présence, suivant une ligne d'univers » (p. 254). On aura deviné que le premier point, là où le peintre doit exprimer le souffle vital et unificateur, correspond à la formule "SAS" » : dans le western de l'âge d'or d'Hollywood, le rôle du shérif est de réunir toutes les forces du bien, et même celles qui s'ignorent (le vieux cowboy alcoolique, la prostituée au cœur tendre, les «Indiens » rebelles, etc.), pour lutter et pour disperser les vraies forces du mal. C'est une sorte de respiration universelle, ou encore ce que les Grecs appelaient la «sympathie ». La situation, l'espace comme unité, le milieu en tant que lignes de force, tous ces éléments sont diffusés à travers les êtres et les poussent à réagir (contre le mal) et à agir (pour faire le bien). Le souffle se contracte dans la situation pour ensuite prendre de l'expansion dans l'action purificatrice.

Sans trop vouloir forcer le parallélisme, on peut reconnaître ici le grand projet réaliste et unificateur d'un Balzac, pour qui la société est une Nature, une organisation dont le roman doit rendre compte. Chez Proust, du moins dans une grande partie de la Recherche, le procédé est plutôt contraire et correspond ainsi à l'autre formule: le souffle 
unitaire est remplacé par des lignes de fuite, des correspondances temporelles dispersées. Ainsi fonctionnent les réminiscences : par exemple la ligne d'univers qui, en dépit de l'invraisemblance de la situation, va relier la madeleine à Combray, la bottine à la mort de sa grand-mère, la raideur d'une serviette au début d'une journée sur la plage à Balbec. Les réminiscences éparses qui peuplent le roman n'ont en rien la volonté d'unifier le récit. Le souvenir pur dévoile plutôt la réalité romanesque du monde, de la même façon que les fictions de la petite forme se reconnaissent en tant que fictions. La ligne d'univers propre à la petite forme est en mesure de relier tous les êtres séparés, sans pour autant dévoiler la raison derrière un tel processus - ce sera le rôle des découvertes esthétiques et ontologiques de "L'adoration perpétuelle», passage du roman qui représente à nos yeux la vraie dimension balzacienne de la Recherche, au-delà des "retours" de personnages. Il faudra donc près de 3000 pages au narrateur pour savoir que faire avec les réminiscences qui le hantent et qui lui présentent des fragments d'une autre vision de son être. On part d'un élément et, de proche en proche, on en découvre d'autres, chacun d'eux étant une fenêtre ouvrant sur un côté de la situation incommensurable, incroyable presque. Dans le cas du narrateur proustien : être oisif toute sa vie et malgré tout avoir accumulé les matériaux pour devenir un grand écrivain.

En espérant que le lecteur nous autorise à continuer notre généalogie intermédiatrice - et par le fait même incomplète - des figures propres aux fictions du vide, nous souhaiterions dire deux mots d'un roman contemporain de la Recherche et qui présente l'avantage d'être lui aussi la biographie (plus ou moins) fictive d'un artiste de génie. Il s'agit du Docteur Faustus, écrit par Thomas Mann, exilé aux États- 
Unis, entre 1943 et 1947, et sous-titré La Vie du compositeur allemand Adrian Leverkühn racontée par un ami. L'ami en question, Serenus Zeitblom, le narrateur du récit (et alter ego de Mann), entame cette biographie quelques années après la mort du compositeur. La biographie, avec ses 600 pages et ses dizaines de chapitres, tente de raconter l'inracontable: la formation d'un génie. Le génie - et son équivalent, la folie est un parfait sujet de petite forme, en ce sens que le génie est précisément ce qui ne s'explique pas, ce qui ne peut pas se dire directement. Qu'on nous comprenne bien: Mann n'est pas Goethe (le corps à corps entre les deux romanciers est même assez violent, entre autres lors de la réécriture du pacte faustien), sa mise en scène du génie et de la folie n'a à proprement parler rien de « romantique ». C'est un autre mythe qui intéresse Mann et qui le pousse à développer une fiction originale, dont la forme s'accorde parfaitement avec le contenu.

Puisque écrire le génie reviendrait à le nier, chacun des chapitres du biographe doit ainsi se borner à n'être qu'un point de vue sur le génie - inexplicable par nature - d'Adrian. La situation est bien incommensurable, inatteignable même. L'intérêt du roman de Mann est précisément d'intégrer ces réflexions sur l'écriture, par le biais de son narrateur intradiégétique, à même la biographie du génial compositeur. La biographie doit donc s'afficher spéculairement en tant que texte littéraire pour, dans le même temps, tenter de mettre en perspective le vertige par lequel la situation initiale se définit. On citera, presque au hasard, un passage au début du texte :

Ici je m'interromps avec le sentiment mortifiant d'avoir commis une bévue artistique, une maladresse. Adrian, lui, n'aurait pas, dans une symphonie, introduit si prématurément un thème pareil. Tout au plus l'eût-il laissé pressentir de façon finement 
voilée, à peine perceptible. D'ailleurs, ce qui vient de m'échapper fera - que sais-je? - au lecteur aussi l'effet d'une allusion obscure, douteuse, et paraît peut-être à mes yeux seuls une indiscrétion et une manière balourde d'enfoncer la porte. Pour un homme comme moi, il est très difficile, il semble presque frivole d'assumer à l'égard d'un sujet qui lui est cher comme la vie et le brûle jusqu'aux moelles, le point de vue de l'artiste, du créateur, et de le traiter comme il l'eût fait, avec une compétence désinvolte. De là ma digression intempestive sur la différence entre le génie pur et l'impur, distinction que je reconnais tout en me demandant si elle est fondée. En fait, l'événement m'a forcé à méditer ce problème avec tant d'application et d'intensité que parfois j'eus le sentiment effroyable d'être ravi hors du plan spirituel qui m'est dévolu et approprié, et d'éprouver moi-même une exaltation «impure » de mes facultés naturelles. (2009, p. 19)

Sans être dans la légèreté comique des films de Lubitsch, il ne s'agit pas non plus d'un tragique comme on a pu le voir chez Nichols. Notre enquête, se transformant en investigation littéraire, nous montre comment la petite forme est elle-même en mesure de s'altérer, ce qui du même coup souligne la capacité d'adaptation des fictions du vide.

Le tragique est ici l'élément génétique du génie : initiation nietzschéenne à la maladie (Adrian attrapant volontairement la syphilis en fréquentant la prostituée Esméralda), désintégration morale (le compositeur, comme Faust bien sûr, aurait conversé avec le diable et lui aurait vendu son âme au profit de la connaissance), etc. La fable romanesque de Mann critique ainsi la «vanité du visible» (p.31), au profit des signes et de l'énigme, figures importantes pour l'instauration fictionnelle du vide. Est également traité le problème de la coexistence de la vie animée et de la vie inanimée, en cela que la psychologie humaine atteint ses limites en même temps que les moindres objets, à commencer par les objets absents, gagnent la fonction d'indice, de marque ou de 
symbole. L'inspiration romantique est jugée vétuste, en comparaison à l'idée subite et imperceptible, ce que Proust nous enseignait déjà avec son art des réminiscences.

Ce qui compte dans la biographie du compositeur Adrian, ce n'est non pas tant ce que nous pouvons en lire, mais l'intervalle qu'il y a entre chacun de ses différents chapitres. La composition de Mann a elle aussi tout d'un circuit disloqué. Le chapitre commence trop tard et se termine trop tôt. Pour paraphraser Rimbaud: le génie est ailleurs. La répétition des intervalles repousse de plus en plus loin la situation, à travers un obscurcissement de la raison qui sous-tend le génie d'Adrian. Au circuit des indices qui sont révélés par les diverses actions narrées (enfance du compositeur, ses années d'étude en théologie, sa rencontre avec la prostituée, etc.) s'en trame un second : celui des écarts. Le dit et le non-dit, plutôt que d'être placés en opposition, forment deux séries parallèles et complémentaires : la biographie s'écrit alors même qu'elle ne cesse de repousser ce qui devrait pourtant en composer le sujet. Tout fragment, qu'il soit dit, suggéré ou simplement imaginé, est bon pour devenir un indice pointant vers la situation infiniment plus grande qui est celle du génie et de la démence $\mathrm{du}$ compositeur Adrian inventeur fictionnel de la musique dodécaphonique. Et c'est bien là un autre des mérites du roman de Mann que d'avoir su associer la petite forme romanesque à la découverte d'une construction musicale contemporaine et novatrice: la musique sérielle, théorisée par Schoenberg au cours des années 1920. Selon notre souhait, on assiste à nouveau à une parfaite adéquation entre le contenu et la forme du roman, en cela que la fiction se construit grâce à ses intervalles, ses atonalités et ses séries. 
Sous le mode de l'ellipse, la biographie mannienne cherche après coup une explication pour le génie du compositeur, pour le caractère inédit de sa musique. Évidemment, cela échoue. La fiction littéraire du vide doit se contenter de restituer l'ambiguïté originaire. Mais Mann va plus loin, il va l'ériger en système. Il fait du vide, de l'intervalle et de l'ellipse les figures substantielles d'une nouvelle forme de biographie et par là-même, de roman. Comme le suggère la petite forme du Docteur Faustus, l'instant biographique n'est plus subordonné à la vraisemblance. Sa tâche est à la fois plus humble et, qui sait, plus importante : il doit être un élément créateur de variations, c'est-à-dire assurer la ronde du circuit des indices et des intuitions.

Proscrits dans la logique de la grande forme, mais encensés dans la redistribution des valeurs telle que promue par les figures du vide, la non-vérité et l'indécidable se voient accordé le statut de composantes créatrices, grâce à cette nouvelle vision de l'œuvre d'art où le vide n'est pas synonyme de «rien», pas plus que le "non » se contente d'être une simple négation. La mise en scène du vide permet aux fictions de devenir des fictions pensantes, à leurs risques et périls. Voilà une découverte qui n'est pas sans réjouir l'enquêteur et le scénariste, qui en profitent même pour retrouver le confort de leur foyer.

\section{L'impossible fin de l'enquête}

Bien malin celui qui croirait résumer cette enquête. Plus encore celui qui se proposerait de la conclure. Que le lecteur n'aille toutefois pas croire que le mérite nous revienne, puisque nous ne sommes qu'un pion parmi tant d'autres sur l'échiquier de ce 
jeu fictionnel. Dans le cas présent, le scénariste n'est pas extérieur à son récit, et les êtres de son histoire ne sont pas condamnés à une existence de papier. Risquons-nous tout de même à formuler quelques remarques générales sur le cours d'action que nous avons décidé de suivre dès l'ouverture de ce que nous appelons, peut-être à tort, une enquête.

Même s'il est sans doute un peu tard pour jouer cette main, le lecteur doit savoir que l'enquête ne s'est pas menée toute seule, sans guide. Au cours de notre filature des intuitions deleuziennes, une autre silhouette n'a cessé de guetter notre cheminement: celle de Bruno Latour. Si notre texte se veut si ardemment une "enquête", c'est d'abord en regard de la méthode constitutive de cet hapax conceptuel qu'est le récent ouvrage Enquête sur les modes d'existence. Bien que celui qui porte le triple chapeau d'anthropologue, de sociologue et de philosophe n'y consacre à proprement parler qu'un chapitre, sa pensée sur la fiction, riche de celles d'Étienne Souriau et de Deleuze, nous a encouragé à mettre en scène nos propres pressentiments: la nécessité inconditionnelle qui consiste à suivre la complexité de l'action.

Loin d'avoir été un "cadre » théorique pour la présente réflexion, on peut se risquer à avancer que la pensée latourienne encourage malgré tout à respecter l'hétérogénéité, à travers cette multiplication des formes d'existence souhaitée par l'auteur. Dans ses ouvrages sur la sociologie, sur l'anthropologie, sur les techniques, sur l'économie ou sur le droit, Latour, de loin, guette la fiction, et plus particulièrement les théoriciens de la fiction. Il leur envie leur souplesse, faisant de l'interprétation une gymnastique. Travailler les fictions devrait nous donner une liberté de mouvement. 
Cette idée latourienne a donc été à la base de notre réflexion, qui se devait de terminer par un mouvement réflexif, sous peine d'incompréhension la plus sévère. Par là-même, le vide nous a semblé l'espace fictionnel le plus adéquat pour acquérir la souplesse nécessaire de tout bon enquêteur, sans oublier la légèreté du scénariste qui sait que son travail n'est qu'une ébauche, car son texte est toujours en devenir. De ce point de vue, la différence de nature qui sépare les fictions cinématographiques des fictions littéraires n'est pas à ce point incommensurable, à l'instar du café sans crème et du café sans lait qui coexistent sur la même table virtuelle.

Pas moins que leurs consœurs littéraires, les fictions cinématographiques incarnent un espace-temps où peuvent se pratiquer les arabesques les plus audacieuses. Pour cette raison, il ne sera pas vain de rendre à Bazin ce qui lui revient, alors que nous l'avons jusqu'ici tenu au bout d'un bâton rhétorique, puisque notre scénario d'enquête avait besoin de son personnage de vilain. En un mot, offrir sans regarder à Bazin le titre de métaphysicien, comme le fait par exemple Alain Badiou, est un geste assez lourd de conséquences. On semble donner un noble titre au critique, au même moment qu'on lui enlève sa liberté de mouvement, pourtant significative lorsqu'on porte une attention, même divagante, à ses nombreux articles qui font œuvre. Certes, on ne peut pas nier que l'intérêt de Bazin est d'avoir su penser la science de l'être cinématographique (de là son "ontologie») en y incluant le moment caractéristique de l'enregistrement. Ce qui est aujourd'hui une évidence ne l'était sans doute pas du vivant de l'auteur. Pour le dire autrement, Bazin a su chercher la poésie de l'enregistrement, faisant de lui ce que Gilbert Simondon appelait à peu près au même moment un « poète technique ». 
Bazin est donc encore célèbre aujourd'hui pour cette révolution de la prise en compte ontologique de l'art à même le procédé. Mais l'auteur de Qu'est-ce que le cinéma? est aussi un brillant acrobate, ne serait-ce que par son intuition, maintes fois répétée, qu'il faut faire dialoguer le cinéma, et plus particulièrement son devenir ontologique, avec celui des autres arts. Une tendance se remarque au sein de cette prospection utopique bazinienne: celle de l'évolution du cinéma vers la pureté, qui est à la fois critiquable et pourtant remarquable. Bien sûr, Bazin a beaucoup théorisé ce qu'il nomme le « cinéma impur », par exemple les adaptations littéraires ou, à la limite, un certain théâtre filmé. Mais si le cinéma s'altère, c'est pour mieux se retrouver, le temps venu. L'impureté bazinienne, de cette manière, serait un autre exemple de gymnastique latourienne : chaque forme d'art aurait son métalangage, que le cinéma en s'altérant s'offre le luxe d'expérimenter, d'interpréter même, afin de peaufiner son propre métalangage.

La littérature, au moins depuis Thomas More, l'inventeur du terme, est à même de produire des utopies. Afin d'éclaircir cette remarque conclusive, on soulèvera une des définitions que propose Pierre Macherey - philosophe de la fiction et de la souplesse interprétative - de ce qu'est justement l'utopie: «l'utopie, forme de pensée en mouvement constant, est inséparable de son bougé, qui, en même temps qu'il a déplacé les cibles, a modifié les procédures par lesquelles elle entreprenait de les atteindre » (2011, p. 12). Une question se pose: ce que l'on caractérise parfois comme l'hégélianisme bazinien - son désir de grande synthèse historique et métaphysique - est-il, dans cette optique bien précise, autre chose qu'une utopie? En effet, le « devenir pur » (l'expression n'est pas de Bazin) du cinéma est inséparable de son 
mouvement, de son bougé, dit bien Macherey, qui l'amène sur les chemins bifurquant de "l'impur». Concevoir Bazin comme un penseur de l'utopie plutôt que comme un penseur de l'ontologie nous paraît être le saut qualitatif nécessaire pour mettre l'accent sur toute l'élasticité qui caractérise son écriture : les différentes rencontres avec l'impur sont autant de dislocations de l'action, comme nous l'ont appris les fictions du vide.

Que l'on nous comprenne bien, nous ne prêchons pas ici pour une ouverture absolue de l'interprétation ou de la pensée critique. Les actes de souplesse, même les plus audacieux — par exemple les travaux des enquêteurs de métier comme Pierre Bayard, Yves Citton, Marc Escola ou Stanley Fish -, se font toujours au sein d'un principe, même lointain, de fermeture. L'interprétation, quoi qu'on en dise et aussi librement qu'on l'approche, est un système. Le meilleur terme serait encore une fois latourien: l'interprétation est un réseau ou, plutôt, l'interprétation est l'acte de faire réseau. Les fictions précédemment étudiées nous ont déjà instruit à ce sujet, en soulignant la capacité d'invention et de réflexion que les auteurs injectent dans leur œuvre grâce aux figures du vide. Avec l'œuvre d'art, le vide n'est pas le néant: avec ses différentes manifestations, il trace un réseau, c'est-à-dire un système orienté, qui est le terrain de jeu de l'enquêteur, nom que nous avons choisi de donner à l'interprète.

Une figure du vide, par exemple une ellipse chez Lubitsch, est toujours une figure orientée. Les fictions du vide, et nous terminerons par cette remarque, ne doivent pas être interprétées du point de vue de l'ontologie traditionnelle, mais par rapport au devenir de l'être. C'est pourquoi l'enquête ne peut être complétée, car elle est toujours en devenir. La seule 
chose que l'on puisse faire est de l'arrêter volontairement, d'accepter son incomplétude et sa volonté de rester une ébauche, en espérant qu'un autre enquêteur vienne en combler les blancs, et qu'une autre enquête reprenne.

\section{Bibliographie}

BAZIN, André. (1975), "Montage interdit», dans Qu'est-ce que le cinéma?, édition définitive, Paris, Éditions du Cerf, coll. « 7e art », p. 49-61.

CERISUELO, Marc. (2012), Fondus enchaînés. Essais de poétique du cinéma, Paris, Seuil, coll. « Poétique ».

FLAUBERT, Gustave. (1989), L'Éducation sentimentale [1869], Paris, Pocket, coll. « Classiques ».

DELEUZE, Gilles. (1983), Cinéma 1. L'Image-mouvement, Paris, Minuit, coll. « Critique».

DURAND, Philippe. (1993), Cinéma et montage. Un art de l'ellipse, Paris, Éditions du Cerf, coll. « 7e art».

LATOUR, Bruno. (2012), Enquête sur les modes d'existence. Une anthropologie des modernes, Paris, La Découverte.

LEUTRAT, Jean-Louis. (2009), Un autre visible. Le fantastique du cinéma, Le Havre, De L'incidence éditeur.

MACHEREY, Pierre. (2011), De l'utopie!, Le Havre, De l'incidence éditeur. 
MANN, Thomas. (2009), Le Docteur Faustus. La vie du compositeur allemand Adrian Leverkühn racontée par un ami [1943-1946], trad. Louise Servicen, Paris, Albin Michel, coll. «Le livre de poche».

NEF, Frédéric. (2011), La Force du vide. Essai de métaphysique, Paris, Seuil, coll. «L'ordre philosophique ».

PAMART, Jean-Michel. (2012), Deleuze et le cinéma. L'armature philosophique des livres sur le cinéma, Paris, Kimé.

PROUST, Marcel. 1975, "À propos du "style" de Flaubert» [1920], dans Essais et articles, Paris, Gallimard, coll. «Bibliothèque de la Pléiade ».

\section{Résumé}

Le présent article, sous la double forme d'un scénario et d'une enquête, se veut une réflexion volontairement ouverte sur ce qui sera défini comme les "fictions du vide», sur leurs différents modes d'apparition et sur les diverses figures qu'elles sont en mesure de proposer. Le point de départ de l'enquête se construit depuis la reprise d'une pensée de Deleuze sur ce qu'il nomme la "petite forme » cinématographique, accompagnée d'exemples choisis dans la filmographie de Chaplin, de Lubitsch et de Mike Nichols. Le second moment de l'enquête sera littéraire, afin de suivre la trajectoire intermédiatique des fictions du vide et ainsi de rendre compte de leur capacité d'adaptation. Les œuvres considérées, pour des raisons différentes, seront À la recherche du temps perdu et Le Docteur Faustus. Ce parcours expressément bifurquant sera finalement l'occasion d'interroger la portée des fictions du vide en rapport avec l'acte même qui consiste à les interpréter. 


\begin{abstract}
This article, in the dual form of a scenario and an investigation, is a reflection deliberately open about what will be defined as "fictions of gap", their different modes of occurrence and the various figures that they are able to offer. The starting point of the investigation is taken from Deleuze's thought on what the philosopher calls the cinematographic "small form", with examples chosen from the filmography of Chaplin, Lubitsch and Mike Nichols. Then the investigation will take a literary turn to follow the intermedia path of the fictions of gap and thus to report their ability to adapt. Works considered, for different reasons, will be In Search of Lost Time and Doctor Faustus. This course will eventually bring the opportunity to examine the scope of the fictions of gap in relation to the concept of interpretation as an act.
\end{abstract}

